\title{
Image and Video Restoration with TV/L2-Norm Constraint
}

\author{
Y. Nojima \\ Graduate school of Information Science and Engineering \\ Ritsumeikan University \\ Japan \\ X.H. Han \\ Graduate school of Information Science and Engineering \\ Ritsumeikan University \\ Japan
}

\begin{abstract}
There is a high demand for generating high-quality video and images, which are used for the wide range of applications, such as biometric authentication, medical imaging, and so on. In this paper, we present a video restoration method for generating a high-quality video from a deteriorated or blurred video. Recent researches independently investigate how to estimate the proper kernel from single blurred image and other researchers developed the unique algorithm, which includes time variation of data for restoring a blurred video. Therefore, in this paper we proposed a high-quality video or image restoration method, which combined with these two researches' methods for enhancing restoration performance. Our strategy can be divided into two steps. The first step is kernel estimation from each image frame of blurred video with using Total Variation (TV)/L2-norm as a regularization term. Then, the second step is to recover a high-quality video with algorithm, which considering time variation of adjacent frames. Experimental results show that the recovered high-resolution video and images with our proposed approach can achieve comparable performance than the conventional methods. In addition, our method can visualize how the blurry degree changes in the video.
\end{abstract}

Keywords-image restoration; deblurring; kernel estimation; total variation

\section{INTRODUCTION}

Image restoration is a well-researched topic that has presented a wide variety of solutions for enhancement of images, which have been recently used in biometric applications. For example, it is used to authenticate persons by using a set of images captured with closed-circuit televisions. However, it is difficult to authenticate a person's identity in case the captured images are blurred and lost important features because of object movement or hardware problem. Therefore, to develop an effective authentication technique used in biometric applications, it is essential to recover a highquality image with important features estimated from a set of blurred images.

For recent years, a lot of image reguralizers, such as $\mathrm{L} 1$, L2 or TV, have been analyzed and applied for image restoration methods by the researchers. However, image restoration is considered only one frame, which means it

\author{
Y.W. Chen \\ Graduate school of Information Science and Engineering \\ Ritsumeikan University \\ Japan
}

doesn't consider time series variation between adjacent frames in video data.

In our research, we focus on not only single image restoration but video restoration, which is considered time series variation. The paper is organized as follows. In Section 2, we briefly describe image and video restoration method. Then, Section 3 outlines our proposed strategy including TV/L2-based deblurring method and Section 4 presents experimental results. Finally, we state conclusion and future work in Section 5.

\section{IMAGE RESTORATION}

In this paper, we formulate the image degradation model as

$$
\mathbf{Y}=\mathbf{H X}
$$

Where $\mathbf{X}$ is the unknown sharp image, $\mathbf{Y}$ is the blurred or degraded image we observe, $\mathbf{H}$ is the blurring matrix, which we called Point Spread Function (PSF) or blurring kernel. Image restoration is to obtain the high resolution image $\mathbf{X}$ and blurring matrix $\mathbf{H}$ fromobserved blurred image $\mathbf{Y}$.

In general, image restoration method has two main steps. First step is to estimate blurring kernel $\mathbf{H}$ with the input blurred image $\mathbf{Y}$ and regularizer function. Then, next step is to refine the blurred image with the estimated kernel $\mathbf{H}$ to obtain the clear image. These two steps are iteratively processed with updating $\mathbf{H}, \mathbf{Y}$ alternately. In the kernel estimation phase, we usually use the blurred image features, which are obtained via filtering with Laplacian or Gabor filter for proper kernel estimation. In case of single blurry image, L1/L2-norm is known as a well-performed reguralizer empirically proven in [2]. Figure 1 shows that L1/L2 function has better performance than other norms such as L1, L2.

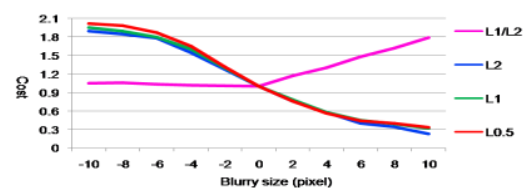

FIGURE I. THE COMPARISON OF L1/L2 AND OTHER IMAGE NORMS [2]. 
In case the image is blurred, the L1/L2 function becomes larger. The cost of L1/L2 gradually attenuates and is stabilized with decreasing the blurry size, which represents the image is sharper or orig inal one. This empirical proof means the L1/L2norm performs better for proper kernel estimation. Therefore, in this paper we also use L1/L2 function as an kernel estimation regularizer.

After estimating kernel, we can use a lot of deconvolution method to restore the sharp image from the blurred image. Richardson-Lucy [4] is one of the most well-known image restoration methods. However, Richardson-Lucy method is sensitive to a wrong kernel, which causes ringing artifacts in the restored image. In addition, this method doesn't consider video data use. Therefore, we apply [3] as deconvolution method. In the nest section, we describe whole process of our proposed strategy.

\section{PROPOSED STRATEGY}

Our proposed strategy consists of two main processes: Kernel estimation with L1/L2-norm and Image/Video restoration with $\mathrm{TV} / \mathrm{L} 2$-norm for recovering the sharp image or video data. Before applying video restoration method to blurred video data, we divide video data to image frames. Then, we can obtain the proper kernel from each blurred frame in the kernel estimation phase with L1/L2-norm. After this process, we apply the time series-variation considered video restoration method with L2/TV-norm [3], which describes in Section 3.2. Through these processes, our proposed method can recover non-blurred high quality video from input blurred one. Our proposed framework is shown in Figure. 2 and procedure of our strategy is described as follows:

Estimate the proper kernel from each blurred image frame with L1/L2-norm constraint

Restore the sharp image/video with L2/TV-norm constraint.

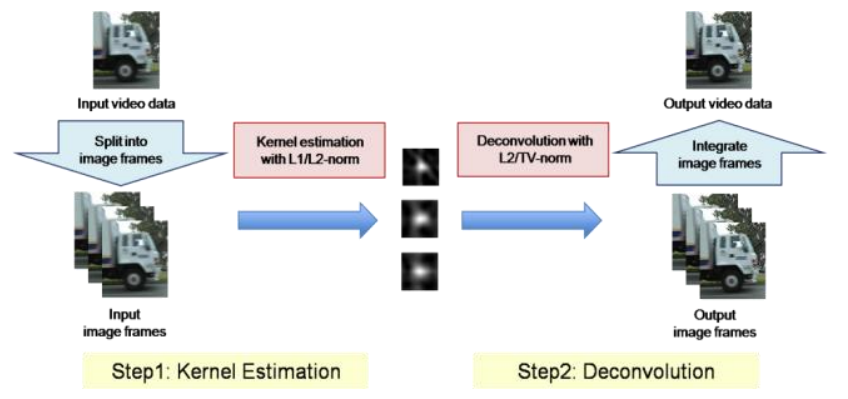

FIGURE II. OUR PROPOSED FRAMEWORK.

Next, we give the detail description of kernel estimation with L1/L2-norm and restoration with L2/TV-norm respectively.

\section{A. Kernel Estimation with Using L1/L2-Norm Constraint}

L1/L2-norm is one of the most effective regularizers for estimating the proper blurry kernel as we described in Section 2. For estimating high accuracy kernel, we use L1/L2-norm in kernel estimation phase [2]. Since we obtain high-frequency feature from the blurred image, we can assume that the estimated kernel and high-frequency component of recovered image are sparse. Therefore, the cost function is formed as:

$$
\min _{x, k} \lambda\|x \otimes k-y\|_{2}^{2}+\frac{\|x\|_{1}}{\|x\|_{2}}+\psi\|k\|_{1}
$$

Where, $x$ and $y$ represent the unknown sharp image and given blurred image in high-frequency space, $k$ is the unknown kernel and $\otimes$ is the 2D convolution operator. The scalar parameter $\lambda$ and $\psi$ control the relative strength between kernel and image regularization. To optimize equation 2 , we can start with initialization on $x$ and $k$, then update respectively with [5].

\section{B. Video Restoration with Using L2/TV-Norm Constraint}

The conventional image restoration method doesn't consider the time series variation. Our propose strategy can apply the blurred video data with using L2/TV-norm [3]. We assume that $\mathbf{f}$ is the unknown video data including the spacetime volume and $\mathbf{H}$ is a linear transformation representing blurring kernel. Then, the cost function of video restoration is formulated as:

$$
\underset{\mathbf{f}}{\operatorname{minimize}} \frac{\mu}{2}\|\mathbf{H f}-\mathbf{g}\|^{2}+\|\mathbf{D f}\|_{1}
$$

Where $\mathbf{g}$ is the observed three dimensional data including space-time volume, $\mathbf{D}$ is an operator calculating the first-order forward finite difference operators along the horizontal, vertical and temporal directions respectively. $\mu$ is a regularization parameter. To solve equation 3 , we first introduce the intermediate variables $\mathbf{u}$ and then transform into equation 4:

$$
L(\mathbf{f}, \mathbf{u}, \mathbf{y})=\frac{\mu}{2}\|\mathbf{H f}-\mathbf{g}\|^{2}+\|\mathbf{u}\|_{1}-\mathbf{y}^{T}(\mathbf{u}-\mathbf{D f})+\frac{\rho_{r}}{2}\|\mathbf{u}-\mathbf{D f}\|^{2}
$$

Where $\rho_{r}$ is a regularization parameter and $\mathbf{y}$ is the Lagrange mu ltiplier. Equation 4 can be divided into three subproblems consist of $\mathbf{f}, \mathbf{y}$ and $\mathbf{u}$. These three sub-problems can be solved iteratively with using TV function and restore the final sharp video or images. Through these steps, our proposed strategy can recover the sharp video data.

\section{EXPERIMENTAL RESULTS}

In this section, we compare the experimental results by our proposed strategy and conventional method [2]. We use the video data captured outside for the experiment. The image size is $480 \times 264$. In our experiment, we set $15 \times 15$ as the maximum size of estimated kernel. Figure 3 shows the experimental results. It is obvious that the detailed components in the image can be well-recovered using our proposed strategy compared to conventional method.

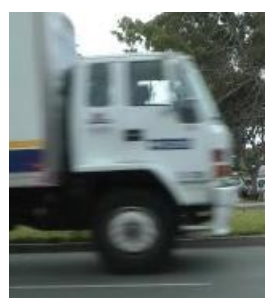

(b) Input

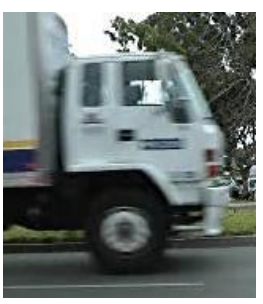

(b) Ours

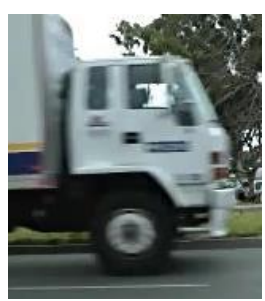

(c) Conventional method [2]
FIGURE III.EXPERIMENTAL RESULT S 


\section{CONCLUSION}

In this study, we proposed a novel image and video restoration method with L2/TV constraint with the blurred image or video data. Experimental results show that our proposed method can obtain well-recovered image than conventional approach and our strategy is effective for real video data since we consider time series variation. However, the restored image by our proposed method has some noises. Thus, in our future work, we will develop the proposed strategy for various type of degradation.

\section{REFERENCES}

[1] Beck, M. Teboulle, "Fast Gradient-Based Algorithms for Constrained Total Variat ion Image Denoising and Deblurring Problems," IEEE Trans. Image Processing, Vol.18 pp. 2419-2434, 2009.

[2] Krishnan Dilip, Terence Tay, and Rob Fergus. "Blind deconvolution using a normalized sparsity measure," Computer Vision and Pattern Recognition (CVPR), 2011 IEEE Conference on. IEEE, 2011

[3] Chan, Stanley H., et al. "An augmented Lagrangian method for total variation video restoration," Image Processing, IEEE Transactions on 20.11, pp. 3097-3111, 2011

[4] Richardson, William Hadley, "Bayesian-Based Iterative Method of Image Restoration," JOURNAL OF THE OPTICAL SOCIETY OF AMERICA, VOLUME 62, NUMBER I, JANUARY, 1972

[5] Fergus, Rob, et al. "Removing camera shake from a single photograph," ACM Transactions on Graphics (TOG). Vol. 25. No. 3. ACM, 2006

[6] S. Cho and S. Lee. "Fast motion deblurring," SIGGRAPH ASIA 2009, 28(5), 2009

[7] Jianping Shi, Li Xu, Jiaya Jia, "Discriminative Blur Detection Feat ures," IEEE Conference on Computer Vision and Pattern Recognition (CVPR), 2014. 